\section{ERRONEOUS ABSCESS}

Sir, the cover of the $B D J$ of 24 January 2014 (volume 216, issue 2), showed a skull with a space occupying lesion in the anterior left maxilla. The caption stated that this lesion was an abscess! An abscess is defined in many dictionaries as a collection of pus. The lesion shown has occupied the anterior left maxilla and expanded and eroded the labial and palatal bone. It is likely to have been a large cyst either apical, residual or developmental, or a benign tumour. It is unlikely to have been a granuloma as that does not reach this large size. An abscess could have arisen in a cyst if it became infected while the subject was alive but this would not have left macroscopic bony evidence. There may be more evidence for the likely pathology on handling the skull than appears on the photograph. The caption also states that the lesion has had an effect on the mandible. There is no evidence for this on the photograph.

I realise that the erroneous caption may have been supplied by the Hunterian Museum of the Royal College of Surgeons but I feel that, since this picture may be used in an educational context, in the interest of accuracy this caption needs to be altered.

\section{B. C. O'Riordan} Watford

DOI: 10.1038/sj.bdj.2014.361
The day highlighted the different perspectives on ARONJ and raised concern that the dental profession on occasion provide inadequate advice for patients receiving antiresorptive therapy with regards to their dental treatment. This letter summarises the key messages from the meeting and is a consensus of the presenters listed below. A multidisciplinary white paper is planned to follow.

In summary the group agreed to support the following:

1. The Scottish Dental Clinical

Effectiveness Programme (SDCEP) -

Oral Health Management of Patients Prescribed Bisphosphonates, Dental Clinical Guidance, April 2011.

2. Improvements in communication between the dentist and the oncologist/haematologist/metabolic bone physicians.

3. Dentists should support the use of antiresorptive therapy (bisphosphonates [BPs] and denosumab) prescribed for patients with osteoporosis and metastatic spread of cancer to bone, and not discourage them from taking the medication due to fear of dental complications. The benefits of these agents clearly outweigh the risks by a wide margin. For patients with osteoporosis, these treatments result in an approximate $60 \%$ reduction in vertebral fractures and 20\% reduction in non-vertebral fractures. In relation to neoplastic disease involving bone, patients benefit significantly with reduction in bone pain, improved quality of life and at least a 50\% reduction in skeletal complications of malignancy including pathological fracture, spinal cord compression, need for radiotherapy and hypercalcaemia.
4. The incidence of ARONJ is extremely low/negligible in patients receiving treatment for osteoporosis (about one in 10,000) and therefore dentists should generally treat these patients as normal dental patients, and routine dental care should be provided. Those patients who have received long-term antiresorptive therapy for osteoporosis should be considered for a more complete risk assessment prior to dentoalveolar surgery given the reporting of persistent, albeit rare, occurrence of ARONJ in this scenario. However, patients at slightly increased risk of ARONJ eg cancer patients receiving higher doses of BPs or denosumab (annual risk about one in 100) should receive regular six-monthly dental care.

5. Any patients receiving high dose potent antiresorptive treatment for cancer (including myeloma) should be referred for dental assessment before starting this therapy when possible (if urgent management precludes this, it should take place as soon as possible). Good communication between the dentist and the oncologist/haematologist is required for optimal management.

6. If dentists encounter patients with ARONJ then they should report the condition using the MHRA yellow forms (http://yellowcard.mhra.gov.uk).

7. The development of a network of dentists for shared experiences, agreed protocols, to encourage reporting, data collection and participation in research is recommended.

T. Renton, T. Taylor, S. Popat,

R. Popat, Z. Sivardeen, I. Fogelman, A. Hawkesford, S. Ruggiero, S. Rogers, S. Leyland, R. Coleman DOI: $10.1038 /$ sj.bdj.2014.360 